\title{
Spatial heterogeneity influences evolutionary scenarios in microbial communities explained by ecological stratification: a simulation study
}

\author{
A.I. Klimenko*, Yu.G. Matushkin, S.A. Lashin \\ Institute of Cytology and Genetics SB RAS, Novosibirsk, Russia \\ Novosibirsk State University, Novosibirsk, Russia \\ *e-mail: klimenko@bionet.nsc.ru
}

Key words: microbial communities, ecological modelling, evolutionary modelling

Motivation and Aim: There are two evolutionary trends in genome organization among bacteria inhabiting microbial communities - towards genome amplification and towards genome reduction. Which scenario overcomes depends on the environmental conditions and the complexity of underlying gene networks. Facing changes of environmental conditions, a population might either adapt or utilize the available reserves more efficiently, or the third way is to migrate pursuing optimal environmental conditions, intruding new habitats and engaging in competition with local species. The impact of such adaptive individual behavior on a developmental scenario of the whole community is of current interest for acquiring a more profound comprehension of interactions between various evolutionary mechanisms.

Methods and Algorithms: The models of evolution of microbial communities were created using the HEC software package [1], which is based on the agent-based approach and super-individual concept [2]. The key simulation unit in the HEC is a genetically homogeneous population of cells that interacts with its environment and other populations. The classification by ecogroups has been proposed that takes into account, the role of organisms in their mutual interactions, and involves their function in the community structure estimating the complexity of their metabolism.

Results: In this simulation study, we have shown that spatially structured habitats allow subdivision of evolutionary trends depending on the distance of a sub-habitat to the nutrient source. We considered ecogroups distributions dynamics according to model parameters. Motile cells typically exhibit the dominance of either aedificators or quasicommensals throughout the whole environment, whereas immobile cells show spatial stratification of dominant ecogroups in line with the distance towards nutrient source. Aedificator biomass prevails in ecosystems consisting of immobile organisms while quasi-mutualist biomass is higher in ecosystems of motile organisms. Active migrations have been shown to impede introduction of new species and to decrease total biomass of the community that is caused by a rapid expansion of well-established populations resulting in the chain reduction of available ecological licenses.

Conclusion: The simulations have shown that ecological patterns of self-organization of microbial communitiescausesustainabilityofdifferentstrategiesunderlyingtheantagonistic evolutionary scenarios. Different evolutionary trends sustain in habitats with contrasting ecological conditions due to nutrient gradients that structure the environment spatially. Acknowledgements: The study was supported by the Budget Project No. 0324-20180017.

\section{References:}

1. Klimenko A.I. et al. (2015) Modeling evolution of spatially distributed bacterial communities: a simulation with the haploid evolutionary constructor. BMC Evol. Biology. 15:S3.

2. Scheffer M., Baveco J.M., DeAngelis D.L., Rose K.A., van Nes E.H. (1995). Ecol Modell. 80:161-170. 